\title{
WEIGHT FUNCTION IN A BIMATERIAL STRIP CONTAINING AN INTERFACIAL CRACK AND AN IMPERFECT INTERFACE. APPLICATION TO BLOCH-FLOQUET ANALYSIS IN A THIN INHOMOGENEOUS STRUCTURE WITH CRACKS*
}

\author{
A. VELLENDER ${ }^{\dagger}$, G. S. MISHURIS ${ }^{\dagger}$, AND A. B. MOVCHAN ${ }^{\ddagger}$
}

\begin{abstract}
We define a weight function in a bimaterial strip containing a semi-infinite crack and an imperfect interface and analyze a problem of antiplane shear. We then present an asymptotic algorithm that uses the weight function to evaluate the coefficients in asymptotics of solutions to problems of wave propagation in a thin bimaterial strip containing a periodic array of cracks situated at the interface between two materials.
\end{abstract}

Key words. Bloch-Floquet waves, boundary layer, crack, imperfect interface, weight function

AMS subject classifications. 35P20, 35Q74, 45E10, 74K10, 74K30

DOI. $10.1137 / 110824838$

1. Introduction. In this paper, we address the problem of determining a weight function in a domain representing a bimaterial strip containing a semi-infinite interfacial crack. Where the crack is not present, the interface is considered imperfect, modeling a thin layer of adhesive between the materials.

Weight functions are mainly used to evaluate stress intensity factors for asymptotic representations near nonregular boundaries such as crack tips. Classically, symmetric weight functions for interfacial cracks in two-dimensional elasticity were studied by Hutchinson, Mear, and Rice [11] and Bueckner [8]. In these classical works, weight functions were defined as the stress intensity factors corresponding to the point force loads applied to the faces of the crack. More recently, Willis and Movchan [22] defined general weight functions as nontrivial singular solutions of a boundary value problem with zero tractions on the faces of the crack and unbounded elastic energy. Recently, weight functions have been used to perform perturbation analysis of the crack front in [20] and to evaluate Lazarus-Leblond constants in [19]. These works contain perfect interfaces that lead to the well-known square root singularity phenomenon [13], [21], [22]. In the imperfect interface problem considered in the present paper, there is no square root singularity in stress components, and so the weight function instead takes the role of aiding in the evaluation of important asymptotic constants that take the place of stress intensity factors.

The imperfect interface is a crucial feature of the problem discussed. Accurate asymptotic derivations with various interfaces in composite materials (of imperfect type among others) for antiplane shear without the presence of cracks have been analyzed in [7], [10], [14]. Such interfaces have been used to model a thin layer consisting of small cracks in such a way that the cracks do not appear in the analysis in [5], [6], [9] using the phenomenological approach. Cracks in the static regime with imperfect interfaces have been studied in [2], [15], where it is proved that the imperfect interface leads to

*Received by the editors February 22, 2011; accepted for publication (in revised form) August 1, 2011; published electronically October 20, 2011.

http://www.siam.org/journals/mms/9-4/82483.html

'Institute of Mathematics and Physics, Aberystwyth University, Ceredigion, SY23 3BZ, UK (asv09@ aber.ac.uk, ggm@aber.ac.uk). The first author received support from Aberystwyth University, which provided APRS funding. The second author received support from the European Union Seventh Framework Programme under contract PIAP-GA-2009-251475.

Department of Mathematical Sciences, University of Liverpool, Liverpool, L69 3BX, UK (abm@liv.ac.uk).

1327

Copyright $@$ by SIAM. Unauthorized reproduction of this article is prohibited. 
a different type of singularity near the crack tip than in the ideal interface case. Analysis of the perfect interface with cracks under harmonic load can be found in [1], [17] and recently for a layered composite with cracks in [9]. The manuscript [17] considers wave propagation in a thin bimaterial strip and discusses the singular behavior near the crack tip, while [1] considers a bimaterial plane with waves propagating perpendicular to the cracks.

We consider in this paper mode III deformation and describe the extent of the interface's imperfection by a positive parameter denoted $\kappa$. The problem we study here is a singular perturbation problem; taking very small values for $\kappa$ gives a qualitatively significantly different weight function from that derived for the perfect interface case in [17], which corresponds to the formulation with $\kappa=0$. Moreover, large values of $\kappa$ can lead to interesting effects, where the boundary layers surrounding different crack tips decay slowly so they can no longer be considered as having no influence on the Bloch-Floquet conditions. This effect is discussed in [4]; in the analysis presented in the present paper, we assume that $\kappa$ is not large enough for these effects to come into play and later find a condition for this to be the case. Problems regarding cracks in domains including imperfect interfaces have been studied in [2] and [16], but no corresponding weight function has previously been constructed.

Another critical characteristic of the problem is that the strip considered is very thin. In addition to the strip itself being very thin, imperfect interfaces are typically replaced with an extremely thin layer of a softer bonding material in finite-element computations (justified, for example, in [7], [10], [16]). Moreover, singular behavior exists at the crack tips. These points make finite element method (FEM) modeling for particularly thin strips extremely difficult or even impossible and motivate the need for the asymptotic approach. In this paper, we compare the asymptotic model with finite-element simulations only in cases when the strip is not too thin, but stress that the finite-element methods are unsuitable for the limiting case, whereas the asymptotics remain valid. The asymptotic method also obtains crucial constants that describe the solution's behavior at the crack tips which are vital for determining whether fracture may occur. These important constants would not be attained by finite-element methods.

The plan of the work is as follows. We first formulate the weight function problem and use Fourier transform and Wiener-Hopf techniques [18] to obtain the solution. Asymptotic analysis enables us to find analytic expressions for all important constants. We then present an application of the weight function to the analysis of Bloch-Floquet waves in a structure containing a periodic array of cracks and imperfect interfaces. This application involves the derivation of junction conditions. Asymptotic theories for structures such as rods and plates have received much attention throughout the history of elasticity theory. For multistructures, however, conditions in engineering practice are often formulated on the basis of intuitive physical assumptions [3]. For example, the zero order junction conditions for the problem addressed fit with physical intuition. It is important to give these conditions a rigorous mathematical footing; moreover, higher order junction conditions do not follow such intuition [12].

We conclude by presenting a comparison between the perfect interface case studied in [17] and the imperfect interface case presented here.

\section{Weight function.}

2.1. Formulation of the problem. The geometry of the strip in which we construct the weight function is shown in Figure 2.1. We define our domain $\Pi_{B}$ to be the union of $\Pi_{B}^{(1)}$ and $\Pi_{B}^{(2)}$, where

Copyright () by SIAM. Unauthorized reproduction of this article is prohibited. 


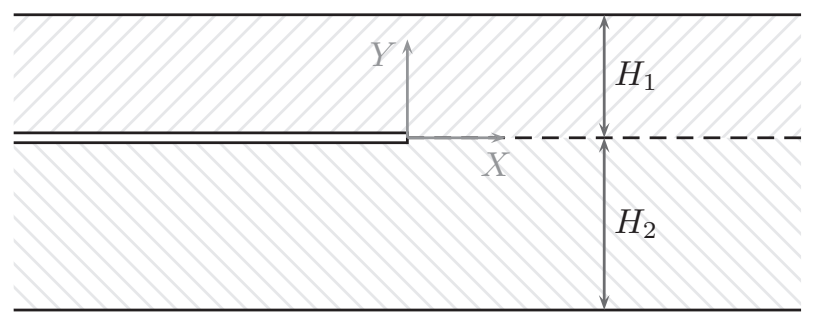

FIG. 2.1. Geometry for the weight function.

$$
\Pi_{B}^{(j)}=\left\{(X, Y): X \in \mathbb{R},(-1)^{j+1} Y \in\left(0, H_{j}\right)\right\}, \quad j=1,2 .
$$

$\Pi_{B}^{(1)}$ corresponds to the material above the cut with shear modulus $\mu_{1}$, while $\Pi_{B}^{(2)}$ corresponds to the material below the cut with shear modulus $\mu_{2}$. The materials have respective thicknesses $H_{1}$ and $H_{2}$. A semi-infinite crack with its tip placed at the origin occupies $X<0$, while the rest of the interface is assumed to be imperfect (see (2.5) in the text below).

The functions $w_{1}$ and $w_{2}$ are defined in domains $\Pi_{1}$ and $\Pi_{2}$, respectively, as solutions to the Laplace equation

$$
\nabla^{2} w_{j}(X, Y)=0
$$

We impose boundary conditions along the horizontal parts of the boundary of $\Pi_{B}$ and on the crack face itself. We denote the components of stress in the out-of-plane direction by

$$
\sigma_{n z}^{(j)}(X, Y):=\mu_{j} \frac{\partial u^{(j)}}{\partial n}, \quad j=1,2
$$

We assume a zero stress component in the out-of-plane direction along the top and bottom of the strip, as well as along the face of the crack itself:

$$
\begin{gathered}
\sigma_{Y Z}^{(1)}\left(X, H_{1}\right)=0, \quad \sigma_{Y Z}^{(2)}\left(X,-H_{2}\right)=0, \quad X \in \mathbb{R}, \\
\sigma_{Y Z}^{(1)}\left(X, 0^{+}\right)=0, \quad \sigma_{Y Z}^{(2)}\left(X, 0^{-}\right)=0, \quad X<0 .
\end{gathered}
$$

Ahead of the cut we impose the imperfect transmission conditions

$$
\left.w_{1}\right|_{Y=0_{+}}-\left.w_{2}\right|_{Y=0_{-}}=\kappa \sigma_{Y Z}^{(1)}\left(X, 0^{+}\right), \quad X>0,
$$

where $\kappa>0$ is a parameter describing the extent of imperfection of the interface. We further assume continuity of tractions across the interface between the materials

$$
\sigma_{Y Z}^{(1)}\left(X, 0^{+}\right)=\sigma_{Y Z}^{(2)}\left(X, 0^{-}\right), \quad X>0 .
$$

We seek solutions in the class of functions that decay exponentially as $X \rightarrow+\infty$ and are bounded as $X \rightarrow-\infty$ :

$$
w_{j}=O\left(e^{-\gamma_{+} X}\right), \quad X \rightarrow+\infty ; \quad w_{j}=C_{j}+O\left(e^{\gamma_{-} X}\right), \quad X \rightarrow-\infty,
$$

Copyright ( by SIAM. Unauthorized reproduction of this article is prohibited. 
where $\gamma_{ \pm}>0$ and $C_{j}$ are constants to be sought from the analysis. At the vertex of the crack, the solution $w_{j}$ is assumed to be weakly singular, with

$$
w_{1}, w_{2}=O(\ln |X|), \quad X \rightarrow 0 .
$$

Formally, conditions (2.1)-(2.7) are similar to those in [17] if we take $\kappa=0$. However, with $\kappa>0$ the problem is a singular perturbation problem and the behavior described in (2.8) is entirely different.

2.2. An auxiliary problem. We now introduce an auxiliary solution $\mathcal{Y}$ that satisfies the Laplace equation (2.1) along with the boundary and transmission conditions (2.3)-(2.6), but the conditions at infinity and at the vertex of the crack are modified as follows:

$$
\begin{aligned}
& \mathcal{Y}_{j}=O\left(e^{-\gamma_{+} X}\right), \quad X \rightarrow+\infty, \\
& \mathcal{Y}_{j}=C_{j} X+D_{j}+O\left(e^{\gamma_{-} X}\right), \quad X \rightarrow-\infty, \\
& \mathcal{Y}_{j}=\mathcal{Y}_{j}\left(0_{+}, 0\right)+O(X \ln |X|), \quad X \rightarrow 0 .
\end{aligned}
$$

The functions $w$ and $\mathcal{Y}$ are related via the formula

$$
w(X, Y)=\frac{\partial}{\partial X} \mathcal{Y}(X, Y)
$$

Bearing this relationship in mind, we often later refer to $\mathcal{Y}$ as a "weight function" as well as $w$. It is also shown in [15] that as $R \rightarrow 0$,

$$
\mathcal{Y}_{ \pm}=\frac{(-1)^{j} a_{0}^{(\mathcal{Y})}}{\pi \mu_{j}}\left\{\frac{\mu_{1} \kappa \pi}{1+\frac{\mu_{1}}{\mu_{2}}}+\left[1-\ln \left(\frac{R}{b_{0}^{(\mathcal{Y})}}\right)\right] R \cos \theta \pm(\pi \mp \theta) R \sin \theta\right\},
$$

where $\mathcal{Y}_{+}$and $\mathcal{Y}_{-}$represent $\mathcal{Y}_{1}(R, \theta)$ and $\mathcal{Y}_{2}(R, \theta)$, respectively, and $(R, \theta)$ describes the usual polar coordinate system with $\theta \in[0, \pi]$ for $\mathcal{Y}_{1}$ and $\theta \in[-\pi, 0]$ for $\mathcal{Y}_{2}$.

2.2.1. Derivation of Wiener-Hopf equation. We define the Fourier transforms of $\mathcal{Y}_{j}$ as

$$
\overline{\mathcal{Y}}_{j}(\xi, Y)=\int_{-\infty}^{\infty} e^{i \xi X} \mathcal{Y}_{j}(X, Y) d X
$$

The functions $\overline{\mathcal{Y}}_{j}$ are analytic in the strip $S=\left\{\xi \in \mathbb{C}:-\gamma_{+}<\operatorname{Im}(\xi)<0\right\}$, and have a double pole only at the point $\xi=0$, so

$$
\overline{\mathcal{Y}}_{j}(\xi, Y) \sim \frac{1}{\xi^{2}} C_{j}-i \frac{D_{j}}{\xi}+O(1), \quad \xi \rightarrow 0 .
$$

Note that the functions $\overline{\mathcal{Y}}_{j}(\xi, Y)$ can be analytically extended to the strip

$$
\tilde{S}=\left\{\xi \in \mathbb{C}:-\gamma_{+}<\operatorname{Im}(\xi)<\gamma_{-}\right\} .
$$

Let us now introduce [Y] , the jump in $\mathcal{Y}$, defined by

$$
[\mathcal{Y}]=\left.\mathcal{Y}_{1}\right|_{Y=0+}-\left.\mathcal{Y}_{2}\right|_{Y=0-} .
$$

Copyright ( by SIAM. Unauthorized reproduction of this article is prohibited. 
We see from $(2.15)$ that the Fourier transform of the jump $[\mathcal{Y}](X)$ generally speaking has a double pole at the point $\xi=0$.

We introduce the following notation:

$$
\Phi^{-}(\xi)=\overline{[\mathcal{Y}]-\left.\mu_{1} \kappa \frac{\partial \mathcal{Y}_{1}}{\partial Y}\right|_{Y=0+}}=\int_{-\infty}^{0}\left([\mathcal{Y}](X)-\left.\mu_{1} \kappa \frac{\partial \mathcal{Y}_{1}}{\partial Y}\right|_{Y=0+}\right) e^{i \xi X} d X,
$$

where we have taken into account $(2.5)$ or, equivalently, the fact that $[\mathcal{Y}]-$ $\left.\mu_{1} \kappa \frac{\partial \mathcal{Y}_{1}}{\partial Y}\right|_{Y=0+}=0$ for $X>0$. The function $\Phi^{-}(\xi)$ is analytic in the half-plane $\operatorname{Im}(\xi)<0$ and has a double pole at $\xi=0$. Thus it can be analytically extended into the half-plane $\mathbb{C}^{-}=\left\{\xi \in \mathbb{C}: \operatorname{Im}(\xi)<\gamma_{-}\right\}$. We further define the function

$$
\Phi^{+}(\xi)=\left.\mu_{1} \int_{0}^{\infty} \frac{\partial \mathcal{Y}_{1}}{\partial Y}\right|_{Y=0+} e^{i \xi X} d X
$$

and so according to $(2.4), \Phi^{+}(\xi)$ is analytic in the half-plane $\mathbb{C}^{+}=\{\xi \in \mathbb{C}$ : $\left.\operatorname{Im}(\xi)>-\gamma_{+}\right\}$.

We expect that

$$
\Phi^{ \pm}(\xi)=\frac{E_{1}^{ \pm}}{\xi}+\frac{E_{2}^{ \pm} \ln (\mp i \xi)}{\xi}+O\left(\frac{1}{\xi^{2}}\right), \quad \xi \rightarrow \infty
$$

in the respective domain according to (2.11); we later confirm this to be true.

The Fourier transforms of the functions $\mathcal{Y}_{j}$ are of the form

$$
\overline{\mathcal{Y}}_{j}(\xi, Y)=A_{j}(\xi) \cosh (\xi Y)+B_{j}(\xi) \sinh (\xi Y) .
$$

Upon the application of boundary and transmission conditions, expressions relating $A_{j}(\xi)$ and $B_{j}(\xi)$ are found:

$$
B_{j}(\xi)=(-1)^{j} A_{j}(\xi) \tanh \left(\xi H_{j}\right), \quad j=1,2 ; \quad \mu_{1} B_{1}(\xi)-\mu_{2} B_{2}(\xi)=0 .
$$

Moreover, $\Phi^{ \pm}(\xi)$ can be expressed in terms of $A_{j}(\xi), B_{j}(\xi)$.

$$
\Phi^{-}(\xi)=A_{1}(\xi)-A_{2}(\xi)-\mu_{1} \kappa \xi B_{1}(\xi), \quad \Phi^{+}(\xi)=\mu_{1} \xi B_{1}(\xi) .
$$

By applying boundary and transmission conditions, we conclude that the functions $\Phi^{+}(\xi)$ and $\Phi^{-}(\xi)$ satisfy the functional equation of the Wiener-Hopf type

$$
\Phi^{-}(\xi)=-\Xi(\xi) \Phi^{+}(\xi)
$$

in the strip $-\gamma_{+}<\operatorname{Im}(\xi)<0$, where

$$
\Xi(\xi)=\frac{1}{\xi}\left(\frac{1}{\mu_{1}} \operatorname{coth}\left(\xi H_{1}\right)+\frac{1}{\mu_{2}} \operatorname{coth}\left(\xi H_{2}\right)+\kappa \xi\right),
$$

and $-\gamma_{+}$is equal to the size of the imaginary part of the first zero of $\Xi(\xi)$ lying below the real axis. We would like to stress that the form of the Wiener-Hopf kernel $\Xi(\xi)$ demonstrates that the weight function problem is a singular perturbation problem as $\kappa \rightarrow 0$; the presence of the term involving $\kappa$ fundamentally alters the asymptotic behavior of $\Xi(\xi)$ as $\xi \rightarrow \infty$.

Copyright (c) by SIAM. Unauthorized reproduction of this article is prohibited. 
2.2.2. Factorization of the Wiener-Hopf kernel. We note that the kernel function $\Xi(\xi)$ as defined in (2.24) can be written in the form

$$
\Xi(\xi)=\kappa \frac{(\lambda+i \xi)(\lambda-i \xi)}{\xi^{2}} \Xi_{*}(\xi)
$$

where

$$
\Xi_{*}(\xi)=\frac{\xi\left(\mu_{1} \operatorname{coth}\left(\xi H_{2}\right)+\mu_{2} \operatorname{coth}\left(\xi H_{1}\right)+\mu_{1} \mu_{2} \kappa \xi\right)}{\mu_{1} \mu_{2} \kappa\left(\lambda^{2}+\xi^{2}\right)}
$$

and

$$
\lambda=\sqrt{\frac{\mu_{1} H_{1}+\mu_{2} H_{2}}{\mu_{1} \mu_{2} H_{1} H_{2} \kappa}}
$$

Now, $\Xi_{*}(\xi)$ is analytic in a strip containing the real axis, clearly positive, even and smooth for all $\xi \in \mathbb{R}$ and has been chosen in such a way so that $\Xi_{*}(\xi)$ tends toward 1 as $\xi \rightarrow \pm \infty$ and as $\xi \rightarrow 0$. Furthermore, the function $\Xi_{*}(\xi)$ can be factorized in the form

$$
\Xi_{*}(\xi)=\Xi_{*}^{+}(\xi) \Xi_{*}^{-}(\xi)
$$

where

$$
\Xi_{*}^{ \pm}(\xi)=\exp \left\{\frac{ \pm 1}{2 \pi i} \int_{-\infty \mp i \beta}^{\infty \mp i \beta} \frac{\ln \Xi_{*}(t)}{t-\xi} d t\right\}
$$

and $\beta>0$ is chosen to be sufficiently small so the contours of integration lie within the strip of analyticity of $\Xi_{*}(\xi)$. The functions $\Xi_{*}^{ \pm}$are analytic in their respective half-planes. To conclude this subsection, we have factorized $\Xi(\xi)$ in the form given in (2.25) and (2.28), where $\Xi_{*}^{ \pm}$are analytic in the half-planes denoted by their superscripts. Note that in the case $H_{1}=H_{2}$, other factorization has been obtained in [2].

2.2.3. Asymptotic behavior of $\boldsymbol{\Xi}_{*}^{+}$. We now seek asymptotic estimates of $\Xi_{*}^{+}(\xi)$. We first note that for $\xi$ within the strip of analyticity,

$$
\Xi(\xi)=\frac{\eta}{\xi^{2}}+O(1), \quad \Xi_{*}(\xi)=1+O\left(|\xi|^{2}\right), \quad \xi \rightarrow 0, \quad \eta=\frac{1}{\mu_{1} H_{1}}+\frac{1}{\mu_{2} H_{2}}
$$

Let us now consider more accurately the behavior of $\Xi_{*}(\xi)$ for $\xi \in \mathbb{R}$ as $\xi \rightarrow \infty$. Noting that $\Xi_{*}(\xi)$ is an even function, it follows from (2.24) that

$$
\Xi_{*}(\xi)=1+\frac{\mu_{1}+\mu_{2}}{\mu_{1} \mu_{2} \kappa|\xi|}-\frac{\lambda^{2}}{\xi^{2}}+O\left(\frac{1}{|\xi|^{3}}\right), \quad \xi \rightarrow \pm \infty
$$

The same estimate is true for any $\xi$ lying in the strip of analyticity. We further find that

$$
\Xi_{*}^{+}(\xi)=1+\frac{\alpha \xi}{\pi i}+O\left(|\xi|^{2}\right), \quad \xi \rightarrow 0
$$

Copyright (C) by SIAM. Unauthorized reproduction of this article is prohibited. 


$$
\Xi_{*}^{+}(\xi)=1+\frac{1}{\pi i} \frac{\left(\mu_{1}+\mu_{2}\right)}{\mu_{1} \mu_{2} \kappa} \frac{\ln (-i \xi)}{\xi}+O\left(\frac{1}{|\xi|}\right), \quad \operatorname{Im}(\xi) \rightarrow+\infty
$$

the derivation of these expressions is given in Appendix A. Here we have defined the asymptotic constant

$$
\alpha=\int_{0}^{\infty} \frac{\ln \Xi_{*}(t)}{t^{2}} d t
$$

The important expression (2.33) describing logarithmic asymptotics at infinity is needed later for (2.43).

2.2.4. Solution of the Wiener-Hopf equation. The factorized equation (2.23) is of the form

$$
-\kappa(\lambda-i \xi) \Phi^{+}(\xi) \Xi_{*}^{+}(\xi)=\frac{1}{\lambda+i \xi} \xi^{2} \Phi^{-}(\xi) \frac{1}{\Xi_{*}^{-}(\xi)} .
$$

Both sides of (2.35) represent analytic functions in the strip $-\gamma_{+}<\operatorname{Im}(\xi)<\gamma_{-}$. Moreover, we now have asymptotic estimates for $\Xi_{*}^{ \pm}(\xi)$ at the zero point in (2.32) and for $\xi \rightarrow \pm \infty$ in (2.33). We deduce that since both sides of (2.35) exhibit the same behavior at infinity in their respective domains according to (2.19), both sides must be equal to a constant, which we denote $\mathcal{A}$. We can therefore obtain explicit expressions for $\Phi^{ \pm}$, which are as follows:

$$
\Phi^{+}(\xi)=-\frac{\mathcal{A}}{\kappa(\lambda-i \xi) \Xi_{*}^{+}(\xi)}, \quad \Phi^{-}(\xi)=\frac{\mathcal{A}(\lambda+i \xi) \Xi_{*}^{-}(\xi)}{\xi^{2}} .
$$

We deduce that

$$
\overline{\mathcal{Y}}_{j}(\xi, Y)=-\frac{\mathcal{A} \Phi^{+}(\xi)}{\mu_{j} \xi}\left\{\frac{\cosh \left(\xi\left(Y+(-1)^{j} H_{j}\right)\right)}{\sinh \left(\xi(-1)^{j+1} H_{j}\right)}\right\}, \quad j=1,2
$$

This allows us to investigate the behavior of $\overline{\mathcal{Y}}_{j}$ as $\xi \rightarrow \pm \infty$ and at the zero point. It also enables us to find the hitherto unknown real constants $C_{j}$ and $D_{j}$.

2.2.5. Evaluation of constants $\boldsymbol{C}_{\boldsymbol{j}}, \boldsymbol{D}_{\boldsymbol{j}}, \boldsymbol{a}_{\mathbf{0}}^{(\mathcal{Y})}, \boldsymbol{\gamma}_{ \pm}$. In this subsection, we evaluate the constants $\gamma_{+}$(defined in $\left.(2.9)\right), \gamma_{-}, C_{j}, D_{j}$ (defined in (2.10)), and $a_{0}^{(\mathcal{Y})}$ (defined in (2.13)). We see from our expressions for $\mathcal{Y}_{j}$ and $\Phi^{+}((2.36)$ and (2.37)), along with our asymptotic estimate for $\boldsymbol{\Xi}_{*}^{+}(\xi)$ as $\xi \rightarrow 0$, that

$$
\overline{\mathcal{Y}}_{j}(\xi)=\frac{(-1)^{j+1} \mathcal{A}}{\kappa \lambda \mu_{j} H_{j}}\left(\frac{1}{\xi^{2}}-\frac{i}{\xi}\left(-\frac{\alpha}{\pi}-\frac{1}{\lambda}\right)\right)+O(1), \quad \xi \rightarrow 0,
$$

where $\alpha$ is the constant defined in (2.34). It follows from our definition of $C_{j}$ and $D_{j}$ in (2.15) that

$$
C_{j}=\frac{(-1)^{j+1} \mathcal{A}}{\kappa \lambda \mu_{j} H_{j}}, \quad D_{j}=\frac{(-1)^{j} \mathcal{A}}{\kappa \lambda \mu_{j} H_{j}}\left(\frac{\alpha}{\pi}+\frac{1}{\lambda}\right)
$$

For normalization, we choose $\mathcal{A}=\kappa \lambda$, giving 


$$
C_{j}=\frac{(-1)^{j+1}}{\mu_{j} H_{j}}, \quad D_{j}=\frac{(-1)^{j}}{\mu_{j} H_{j}}\left(\frac{\alpha}{\pi}+\frac{1}{\lambda}\right)
$$

The chosen normalization leaves the expression for $C_{j}$ in (2.40) in the same form as in [17], but it is clearly seen that the expression for $D_{j}$ (which depends upon $\kappa$ ) is different. Mishuris (2001) [15] demonstrates that near the crack tip (i.e., as $R \rightarrow 0$ ), $\mathcal{Y}_{j}(R, \theta)$ has behavior described by (2.13). From this we see that

$$
[\mathcal{Y}] \sim-\kappa a_{0}^{(\mathcal{Y})}, \quad R \rightarrow 0 .
$$

The imperfect transmission conditions (2.5) therefore give that

$$
\left.\mu_{1} \frac{\partial \mathcal{Y}_{1}}{\partial Y}\right|_{Y=0+} \sim-a_{0}^{(\mathcal{Y})}, \quad X \rightarrow 0
$$

We earlier made an assumption in (2.19) regarding the behavior of $\Phi^{+}$at infinity and now verify that this was correct. It follows from the expression for $\Phi^{+}(\xi)$ given in (2.36) and the asymptotic estimate for $\boldsymbol{\Xi}_{*}^{+}(\xi)$ given in (2.33) that

$$
\Phi^{+}(\xi)=\frac{\lambda}{i \xi}+\frac{\left(\mu_{1}+\mu_{2}\right) \lambda}{\mu_{1} \mu_{2} \pi \kappa \xi^{2}} \ln (-i \xi)+O\left(\frac{1}{|\xi|^{2}}\right), \quad \operatorname{Im}(\xi) \rightarrow+\infty
$$

which justifies our previous claim. Theorem B.1 (using $\mu_{1} \frac{\partial \mathcal{Y}}{\partial Y}$ in place of "f" in the statement of the theorem) then yields that

$$
\lim _{X \rightarrow 0+} \mu_{1} \frac{\partial \mathcal{Y}}{\partial Y}=-\lambda
$$

where $\lambda$ has been defined in (2.27) and so it follows that

$$
a_{0}^{(\mathcal{Y})}=\lambda
$$

The constant $\gamma_{+}$is the distance of the first zero of $\Xi(\xi)$ below the real axis. Manipulation of (2.24) indicates that zeros of $\boldsymbol{\Xi}(\xi)$ satisfy

$$
\frac{1}{\mu_{1}} \cot \left(\gamma_{+} H_{1}\right)+\frac{1}{\mu_{2}} \cot \left(\gamma_{+} H_{2}\right)-\kappa \gamma_{+}=0 .
$$

For the first zero below the axis, for large $\kappa, \gamma_{+}$should be small, and so it can be shown that

$$
\gamma_{+}(\kappa)=\lambda(\kappa)\left(1+O\left(\kappa^{-1}\right)\right), \quad \kappa \rightarrow \infty,
$$

indicating that $\gamma_{+}(\kappa)=O\left(\kappa^{-1 / 2}\right), \kappa \rightarrow \infty$. We also see that

$$
\gamma_{+}(0) \in\left(\frac{\pi}{2 H_{1}}, \frac{\pi}{2 H_{2}}\right) .
$$

The constant $\gamma_{-}$is given by

$$
\gamma_{-}=\pi \min \left\{\frac{1}{H_{1}}, \frac{1}{H_{2}}\right\} .
$$

Copyright ( by SIAM. Unauthorized reproduction of this article is prohibited. 
In conjunction with (2.40), we have now found all constants describing the asymptotic behavior of the weight function $\mathcal{Y}$.

3. Application to analysis of Bloch-Floquet waves. In this section, we present an application of the weight function derived in the previous section by addressing the problem of out-of-plane shear Bloch-Floquet waves within a thin bimaterial strip containing a periodic array of longitudinal cracks and imperfect interfaces. The problem addressed is an imperfect interface analogue to that studied in [17].

3.1. Geometry. The geometry of an elementary cell of the thin periodic structure considered is shown in Figure 3.1. The elementary cell is of length $a$ and contains two materials of thicknesses $\varepsilon H_{1}$ and $\varepsilon H_{2}$, where $\varepsilon$ is a small dimensionless parameter. These materials occupy respective domains $\Pi^{(j)}, j=1,2$, and the elementary cell is further split into smaller domains $\Omega_{\varepsilon}^{(m)}, m=1,2,3,4$, as shown in Figure 3.1. Along the interface of the two materials and centered on the origin sits a crack of length $l$. Outside the crack, the interface is assumed to be imperfect, which models a thin layer of adhesive joining the materials together. The extent of this imperfection is represented by the parameter $\kappa$.

The functions $u^{(j)}(x, y)$ are defined in $\Pi_{\varepsilon}^{(j)}, j=1,2$, as solutions of the Helmholtz equations

$$
\nabla^{2} u^{(j)}(x, y)+\frac{\omega^{2}}{c_{j}^{2}} u^{(j)}(x, y)=0, \quad(x, y) \in \Pi_{\varepsilon}^{(j)}, \quad j=1,2 .
$$

Here, $c_{j}=\sqrt{\mu_{j} / \rho_{j}}$ are the shear speeds in their respective domains $j=1,2$. The functions $u^{(j)}$ are regarded as out-of-plane displacements, $\mu_{j}$ denotes the shear modulus, and $\rho_{j}$ denotes the mass density of the material occupying $\Pi_{\varepsilon}^{(j)}$. The quantity $\omega$ represents the radian frequency of the time-harmonic vibrations with amplitude $u$.

3.2. Boundary conditions. We impose boundary conditions along the horizontal parts of the boundary of $\Pi_{\varepsilon}$ and on the crack face itself. We use similar notation to that in the previous section to denote the components of stress (see (2.2)).

We assume a zero stress component in the out-of-plane direction along the top and bottom of the strip, as well as along the face of the crack itself:

$$
\begin{gathered}
\sigma_{y z}^{(1)}\left(x, \varepsilon H_{1}\right)=0, \quad \sigma_{y z}^{(2)}\left(x,-\varepsilon H_{2}\right)=0, \quad x \in(-a / 2, a / 2), \\
\sigma_{y z}^{(1)}\left(x, 0^{+}\right)=0, \quad \sigma_{y z}^{(2)}\left(x, 0^{-}\right)=0, \quad x \in(-l / 2, l / 2) .
\end{gathered}
$$

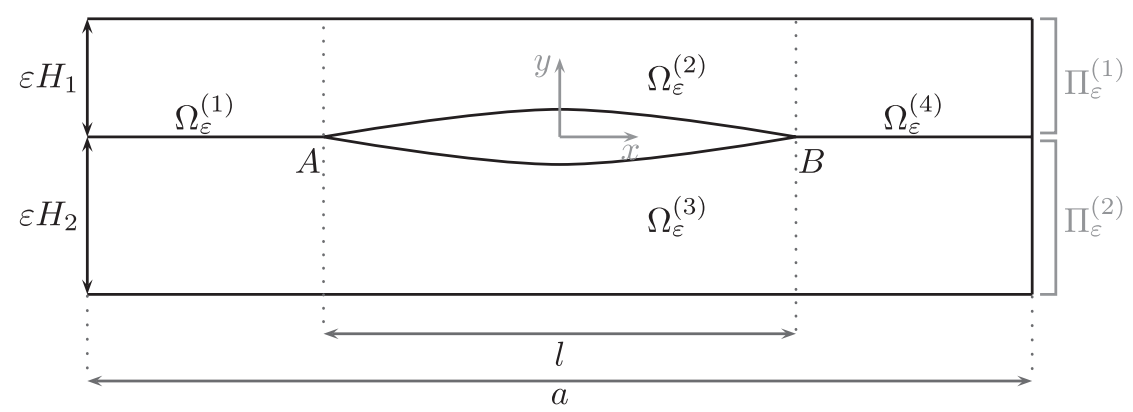

FIG. 3.1. Geometry of the elementary cell.

Copyright ( by SIAM. Unauthorized reproduction of this article is prohibited. 
Outside the crack, along the boundary between $\Pi_{\varepsilon}^{(1)}$ and $\Pi_{\varepsilon}^{(2)}$, there is an imperfect interface described by the condition

$$
u^{(1)}\left(x, 0^{+}\right)-u^{(2)}\left(x, 0^{-}\right)=\varepsilon \kappa \sigma_{y z}^{(1)}\left(x, 0^{+}\right), \quad x \in(-a / 2,-l / 2) \cup(l / 2, a / 2) .
$$

We also assume continuity of stress across the interface

$$
\sigma_{y z}^{(1)}\left(x, 0^{+}\right)=\sigma_{y z}^{(2)}\left(x, 0^{-}\right), \quad x \in(-a / 2,-l / 2) \cup(l / 2, a / 2) .
$$

We seek the solutions $u^{(j)}$ that represent the Bloch-Floquet waves, so that at the ends of our elementary cell $x= \pm a / 2$, we have for $j=1,2$ the Bloch-Floquet conditions

$$
\begin{aligned}
& u^{(j)}(-a / 2, y)=e^{-i K a} u^{(j)}(a / 2, y), \quad y \in\left(-\varepsilon H_{2}, \varepsilon H_{1}\right), \\
& \sigma_{x z}^{(j)}(-a / 2, y)=e^{-i K a} \sigma_{x z}^{(j)}(a / 2, y), \quad y \in\left(-\varepsilon H_{2}, \varepsilon H_{1}\right) .
\end{aligned}
$$

For a fixed value of the Bloch parameter $K$, we seek the eigenvalues $\omega$ and the corresponding eigenfunctions $u^{(j)}$ with finite norm in $W_{2}^{1}\left(\Pi_{\varepsilon}^{(j)}\right), j=1,2$.

In (3.4), the case in which $\kappa=0$ corresponds to an ideal/perfect interface between the different materials; such a problem was considered in [17]. Where possible, we will follow the same line as in that paper. To summarize the approach, we approximate $u$ in a certain form, derive a lower-dimensional model together with boundary layers in the vicinity of the vertices of the crack, and then use our weight function to assist in the derivation of junction conditions for a skeleton model.

3.3. Asymptotic ansatz. The eigenfunctions $u(x, y)$ are approximated in the form

$$
\begin{aligned}
u(x, y, \varepsilon)= & \sum_{k=0}^{N} \varepsilon^{k}\left\{\sum_{m=1}^{4} \chi_{m}\left(v_{m}^{(k)}(x)+\varepsilon^{2} V_{m}^{(k)}(x, Y)\right)\right. \\
& \left.+\left(W_{A}^{(k)}\left(X_{A}, Y\right)+W_{B}^{(k)}\left(X_{B}, Y\right)\right)\right\}+R_{N}(x, y, \varepsilon)
\end{aligned}
$$

with scaled coordinates $X_{A}, X_{B}$, and $Y$ introduced in the vicinity of the left and right vertices of the crack defined as

$$
X_{A}=\frac{x-x_{A}}{\varepsilon}, \quad X_{B}=\frac{x-x_{B}}{\varepsilon}, \quad Y=\frac{y}{\varepsilon} .
$$

Here, $v_{m}^{(k)}$ represent solutions of lower-dimensional problems within limit sets $\Omega_{0}^{(m)}$, $m=1,2,3,4 . \chi_{m}=\chi_{m}(x, y, \varepsilon)$ are cut-off functions defined so that $\chi_{m}(x, y ; \varepsilon) \equiv 1$ in $\Omega_{\varepsilon}^{(m)}$ and decay rapidly to zero outside $\Omega_{\varepsilon}^{(m)}$. They vanish near the so-called junction points $A$ and $B$ (the vertices of the crack). The terms $W_{A}^{(k)}$ and $W_{B}^{(k)}$ represent the boundary layers near $A$ and $B$, and $V_{m}^{(k)}$ is the "fast" change of eigenfunctions in the transverse direction in the domain $\Omega_{\varepsilon}^{(j)}$. $R_{N}$ is the remainder term in the asymptotic approximation. We would like to indicate to the reader that the uppercase scaled coordinate $X_{B}$ defined in (3.9) corresponds to $X$ from the derivation of the weight function in section 2 .

We note that this form of ansatz relies upon the vital assumption that the boundary layers surrounding the crack vertices $A$ and $B$ are independent. That is, we assume that

Copyright ( by SIAM. Unauthorized reproduction of this article is prohibited. 
the exponential decay of both boundary layers is sufficiently rapid so that it is negligible in the vicinity of the other crack tip.

In this paper, we will consider the form of approximation given in (3.8) with $N=1$ and will comment on the effect of taking higher order approximations.

3.4. One-dimensional model problems. Outside the vicinity of $A$ and $B$, the boundary layers $W_{A}^{(j)}$ and $W_{B}^{(j)}$ decay (we later verify this to be the case) and so seek $u$ in the form

$$
u(x, y, \varepsilon) \sim \sum_{k=0}^{1} \varepsilon^{k}\left(v_{m}^{(k)}(x)+\varepsilon^{2} V_{m}^{(k)}(x, Y)\right),
$$

where $V_{m}^{(k)}$ have zero average over the cross-section of $\Omega_{\varepsilon}^{(m)}$ for all $m=1,2,3,4$. That is,

$$
\int_{0}^{H_{1}} V_{m}^{(k)}(x, Y) d Y=0, \quad \int_{-H_{2}}^{0} V_{m}^{(k)}(x, Y) d Y=0 .
$$

Since the low-dimensional model problem studied in [17] was the same above and below the crack (in $\Omega_{\varepsilon}^{(2)}$ and $\Omega_{\varepsilon}^{(3)}$ ), we refer the reader to that paper. The problem is, however, differently formulated in $\Omega_{\varepsilon}^{(1)}$ and $\Omega_{\varepsilon}^{(4)}$ due to the imperfect transmission conditions in these domains. We focus our attention on the layered structure $\Omega_{\varepsilon}^{(1)}$; analogous arguments will apply to $\Omega_{\varepsilon}^{(4)}$. We use the notation $v_{1 j}^{(k)}$ to denote the function $v_{1}^{(k)}$ in $\Pi_{\varepsilon}^{(j)}$. The key observation is then to note that the transmission condition across the imperfect interface as given in (3.4) implies that

$$
v_{11}^{(k)}-v_{12}^{(k)}=0, \quad k=0,1,
$$

and so it follows that for $k=0,1$, the solution to this low-dimensional model is not impacted by the presence of the imperfect interface.

To conclude this section, we have found that our case with the imperfect interface has the same equations for the low-dimensional model up to terms in $\varepsilon$ as the case with the perfect interface studied in [17]. The equations for $v_{4}^{(k)}$ and $V_{4}^{(k)}$ are of course similar to the case examined here, where $m=1$. We would like to stress that the imperfect interface impacts on the low-dimensional model equations for terms in $\varepsilon^{k}, k \geq 2$. The equations gained in this section need to be complemented with the boundary conditions and junction conditions at the points $x_{A}$ and $x_{B}$. In order to derive these junction conditions that depend on the imperfect parameter $\kappa$, we construct boundary layers in the vicinity of the vertices of the crack.

4. Junction conditions. We introduce four smooth cut-off functions $\chi_{m} \in$ $C^{\infty}(\mathbb{R})$ in the spirit of [17]. These are functions defined so that $\chi_{m}(x, y ; \varepsilon) \equiv 1$ in $\Omega_{\varepsilon}^{(m)}$ and decay rapidly to zero outside $\Omega_{\varepsilon}^{(m)}$. These allow us to extend the function (3.10) outside $\boldsymbol{\Omega}_{\varepsilon}^{(m)}, m=1,2,3,4$, giving

$$
u(x, y ; \varepsilon) \sim \sum_{k=0}^{1} \varepsilon^{k} \sum_{m=1}^{4} \chi_{m}(x, y, \varepsilon)\left(v_{m}^{(k)}(x)+\varepsilon^{2} V_{m}^{(k)}(x, Y)\right) ;
$$

however, this gives an error near the junction points $x_{A}$ and $x_{B}$. We therefore introduce boundary layers $W_{A}\left(X_{A}, Y\right)$ and $W_{B}\left(X_{B}, Y\right)$, and so seek $u(x, y, \varepsilon)$ in the form 


$$
u \sim \sum_{k=0}^{1} \varepsilon^{k}\left\{\sum_{m=1}^{4} \chi_{m}\left(v_{m}^{(k)}(x)+\varepsilon^{2} V_{m}^{(k)}(x, Y)\right)+W_{A}^{(k)}\left(X_{A}, Y\right)+W_{B}^{(k)}\left(X_{B}, Y\right)\right\} .
$$

Substituting this expression into the original equation and comparing terms of the same degree of $\varepsilon$, we obtain

$$
\nabla_{X_{\alpha} Y}^{2}\left\{W_{\alpha}^{(k)}\left(X_{\alpha}, Y\right)+\mathcal{F}_{\alpha}^{(k)}\left(X_{\alpha}, Y\right)\right\}=0, \quad \alpha=A, B, \quad k=0,1,
$$

with the functions $\mathcal{F}_{\alpha}^{(k)}, k=0,1, \alpha=A, B$ given by

$$
\begin{aligned}
\mathcal{F}_{A}^{(0)}= & \sum_{m=1}^{3} v_{m}^{(0)}\left(x_{A}\right) \chi_{m}(x, y ; \varepsilon), \quad \mathcal{F}_{B}^{(0)}=\sum_{m=2}^{4} v_{m}^{(0)}\left(x_{B}\right) \chi_{m}(x, y ; \varepsilon), \\
\mathcal{F}_{A}^{(1)} & =\sum_{m=1}^{3}\left\{\left(v_{m}^{(0)}\right)^{\prime}\left(x_{A}\right) X_{A}+v_{m}^{(1)}\left(x_{A}\right)\right\} \chi_{m}(x, y ; \varepsilon), \\
\mathcal{F}_{B}^{(1)} & =\sum_{m=2}^{4}\left\{\left(v_{m}^{(0)}\right)^{\prime}\left(x_{B}\right) X_{B}+v_{m}^{(1)}\left(x_{B}\right)\right\} \chi_{m}(x, y ; \varepsilon) .
\end{aligned}
$$

We now focus our attention near $x_{B}$; analogous arguments apply to $x_{A}$. We will consider in the following analysis four functions $g_{i}, i=1,2,3,4$, which are solutions of the Laplace equation. These solutions also satisfy the boundary conditions corresponding to zero stress on the top and bottom edges of the strip (2.3) as well as along the cut itself (2.4). They also satisfy the transmission condition (2.5) across the imperfect interface, along with continuity of stress (2.6). These solutions are given by

$$
g_{1}=1, \quad g_{2}=X_{B}, \quad g_{3}=\mathcal{Y}, \quad g_{4}=\frac{\partial \mathcal{Y}}{\partial X},
$$

where $\mathcal{Y}$ is the weight function derived in section 2 .

Since they are boundary layers, we expect that $W_{B}^{(k)}$ decay exponentially as $X \rightarrow$ $+\infty$ and behave as $C_{j}^{(k)} X+D_{j}^{(k)}$ as $X \rightarrow-\infty$. We first express $C_{j}^{(k)}, D_{j}^{(k)}, k=0,1$, in terms of $v_{m}^{(k)}$ and their derivatives. We have from Green's formula that

$$
0=\sum_{j=1}^{2} \mu_{j} \int_{\partial \Pi_{B}^{(j)}(L)}\left(g_{i} \frac{\partial}{\partial n}\left(W_{B}^{(k)}+\mathcal{F}_{B}^{(k)}\right)-\left(W_{B}^{(k)}+\mathcal{F}_{B}^{(k)}\right) \frac{\partial g_{i}}{\partial n}\right) d S .
$$

The further analysis is quite similar to that in [17], although we would like to stress that the weight function $\mathcal{Y}$ in the present paper is different, as are the transmission conditions. We therefore need to prepare this analysis from the beginning, where it is different for $g_{3}, g_{4}$.

4.1. The cases $\boldsymbol{k}=\mathbf{0}, \mathbf{1}, \boldsymbol{i}=\mathbf{1}, \mathbf{2}, \mathbf{3}$. We see from boundary conditions that integrals over the horizontal parts of the boundary $l_{1}^{(j)}, l_{3}^{(j)}, l_{4}^{(j)}, j=1,2$, give zero contribution to the integral. Moreover, the contribution from $S_{\delta}$ also disappears as $\delta \rightarrow 0$ (see Figure 4.1) for $g_{1}, g_{2}$, and $g_{3}$, leaving contributions solely from $l_{2}^{(j)}$ and $l_{5}^{(j)}$ in these cases.

From the definitions of $\mathcal{F}_{B}^{(k)}$, we obtain the following limits at $\pm \infty$ for $k=0,1$ :

$$
\mathcal{F}_{B}^{(0)}=v_{4}^{(0)}\left(x_{B}\right), \quad X_{B} \rightarrow+\infty,
$$

Copyright ( by SIAM. Unauthorized reproduction of this article is prohibited. 


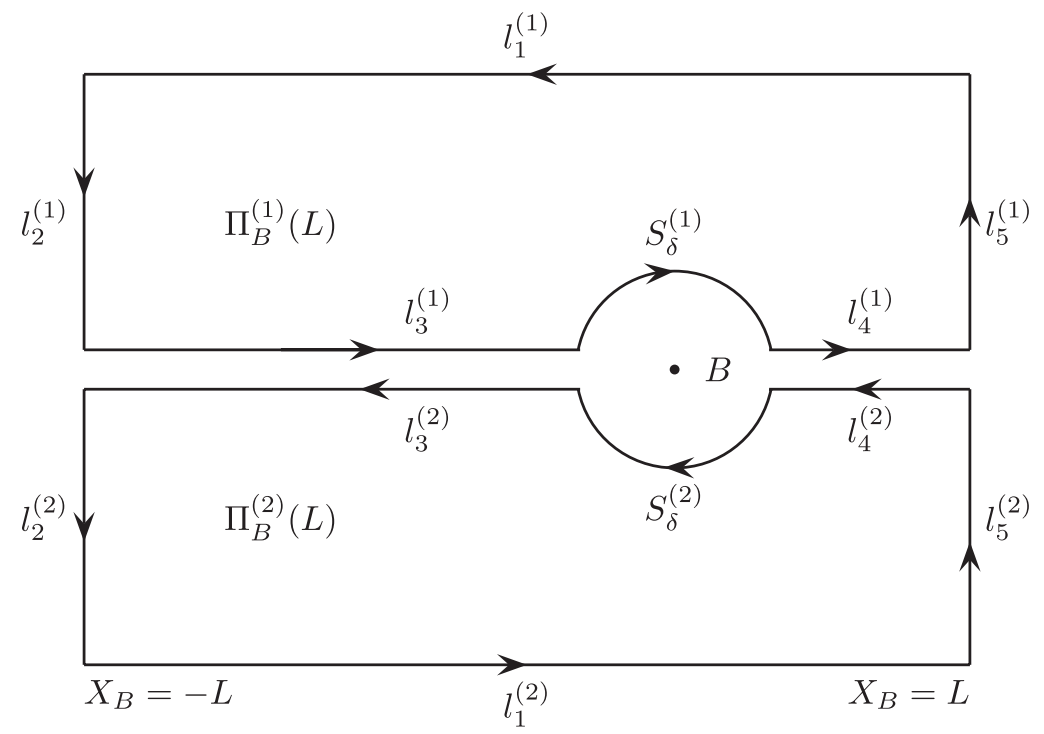

FIG. 4.1. Contour of integration for (4.6).

$$
\begin{aligned}
\mathcal{F}_{B}^{(0)} & =v_{2}^{(0)}\left(x_{B}\right) \mathcal{H}(Y)+v_{3}^{(0)}\left(x_{B}\right) \mathcal{H}(-Y), \quad X_{B} \rightarrow-\infty, \\
\mathcal{F}_{B}^{(1)} & =\left(v_{4}^{(0)}\right)^{\prime}\left(x_{B}\right) X_{B}+v_{4}^{(1)}\left(x_{B}\right), \quad X_{B} \rightarrow+\infty, \\
\mathcal{F}_{B}^{(1)} & =\sum_{j=2}^{3}\left\{\left(v_{j}^{(0)}\right)^{\prime}\left(x_{B}\right) X_{B}+v_{j}^{(1)}\left(x_{B}\right)\right\} \mathcal{H}\left((-1)^{j} Y\right), \quad X_{B} \rightarrow-\infty,
\end{aligned}
$$

where $\mathcal{H}(Y)$ is the Heaviside step function. Since $W_{B}^{(k)} \rightarrow 0$ as $X_{B} \rightarrow+\infty$, (4.6) reduces to

$$
\begin{aligned}
0= & \sum_{j=1}^{2} \mu_{j} \int_{l_{5}^{(j)}}\left(g_{i} \frac{\partial}{\partial X_{B}} \mathcal{F}_{B}^{(k)}-\mathcal{F}_{B}^{(k)} \frac{\partial g_{i}}{\partial X_{B}}\right) d S \\
& -\sum_{j=1}^{2} \mu_{j} \int_{l_{2}^{(j)}}\left(g_{i} \frac{\partial}{\partial X_{B}}\left(\mathcal{F}_{B}^{(k)}+W_{B}^{(k)}\right)-\left(\mathcal{F}_{B}^{(k)}+W_{B}^{(k)}\right) \frac{\partial g_{i}}{\partial X_{B}}\right) d S .
\end{aligned}
$$

Applying this procedure with each of $g_{1}, g_{2}, g_{3}$, and $\mathcal{F}_{B}^{(0)}, \mathcal{F}_{B}^{(1)}$ yields six equations, which are presented in subsection 4.3.

4.2. The cases $\boldsymbol{k}=\mathbf{0}, \mathbf{1}, \boldsymbol{i}=\mathbf{4}$. To obtain a further two equations, we apply the same procedure to the solution $g_{4}=\frac{\partial \mathcal{Y}}{\partial X_{B}}$. Again, the contribution from the horizontal parts of the contour of integration is zero, leaving nonzero contributions from the vertical parts of the contour, $l_{2}^{(j)}$ and $l_{5}^{(j)}$. Unlike with $g_{1}, g_{2}$, and $g_{3}$, however, the contribution from $S_{\delta}{ }^{(j)}$ is nonzero. We investigate the behavior of $g_{4}$ near the crack tip.

We have that $g_{4}^{(j)}=\frac{\partial \mathcal{Y}_{j}}{\partial X}=\frac{\partial \mathcal{Y}_{j}}{\partial R} \cos \theta-\frac{1}{R} \frac{\partial \mathcal{Y}_{j}}{\partial \theta} \sin \theta$, where $(R, \theta)$ is the usual polar coordinate system, with $R=\sqrt{X_{B}^{2}+Y^{2}}$, and so from our asymptotic estimate for $\mathcal{Y}_{j}$ near the crack tip, we deduce that near the crack tip,

Copyright ( by SIAM. Unauthorized reproduction of this article is prohibited. 


$$
g_{4}^{(j)} \sim \frac{(-1)^{j}}{\pi \mu_{j}}\left\{b_{0}^{(\mathcal{Y})}+a_{0}^{(\mathcal{Y})} \ln R+(-1)^{(j+1)} a_{0}^{(\mathcal{Y})} \sin 2 \theta\left(\pi+(-1)^{j} \theta\right)\right\},
$$

and so for small $R$,

$$
\frac{\partial g_{4}^{(j)}}{\partial R} \sim \frac{(-1)^{j} a_{0}^{(\mathcal{Y})}}{\pi \mu_{j} R} .
$$

Noting that the outward normal to $S_{\delta}^{(j)}$ is in the direction of $-R$, we have that as $\delta \rightarrow 0$,

$$
\begin{aligned}
& \mu_{j} \int_{S_{\delta}}\left(g_{4}\left(-\frac{\partial}{\partial R}\right)\left(W_{B}^{(k)}+\mathcal{F}_{B}^{(k)}\right)-\left(W_{B}^{(k)}+\mathcal{F}_{B}^{(k)}\right)\left(-\frac{\partial g_{4}}{\partial R}\right)\right) d S \\
= & \mu_{j} \int_{S_{\delta}}\left(\left(W_{B}^{(k)}+\mathcal{F}_{B}^{(k)}\right) \frac{\partial g_{4}}{\partial R}\right) R d \theta=\mu_{j} \int_{S_{\delta}} \frac{(-1)^{j} a_{0}^{(\mathcal{Y})}}{\pi \mu_{j} R}\left(W_{B}^{(k)}+\mathcal{F}_{B}^{(k)}\right) R d \theta .
\end{aligned}
$$

Since $W_{B}$ satisfies the same model problem as $\mathcal{Y}$, it too will possess asymptotic behavior at the crack tip of the same form as $g_{4}$ in (4.13), but with different constants, which we denote $a_{(k)}^{(W)}$ and $b_{(k)}^{(W)}$, for $k=0,1$. The contribution to the integral from the circular part of the contour is therefore given by

$$
\begin{gathered}
-\frac{a_{0}^{(\mathcal{Y})}}{\pi} \int_{0}^{\pi}\left(W_{B}^{(k)}\left(0^{+}, \theta\right)+\mathcal{F}_{B}^{(k)}\left(0^{+}, \theta\right)\right) d \theta+\frac{a_{0}^{(\mathcal{Y})}}{\pi} \int_{-\pi}^{0}\left(W_{B}^{(k)}\left(0^{+}, \theta\right)+\mathcal{F}_{B}^{(k)}\left(0^{+}, \theta\right)\right) d \theta \\
=-\frac{a_{0}^{(\mathcal{Y})}}{\pi} \int_{0}^{\pi} \frac{-1}{\pi \mu_{1}} \frac{\mu_{1} \kappa \pi}{1+\frac{\mu_{1}}{\mu_{2}}} a_{(k)}^{(W)} d \theta+\frac{a_{0}^{(\mathcal{Y})}}{\pi} \int_{-\pi}^{0} \frac{1}{\pi \mu_{2}} \frac{\mu_{1} \kappa \pi}{1+\frac{\mu_{1}}{\mu_{2}}} a_{(k)}^{(W)} d \theta=\kappa a_{0}^{(\mathcal{Y})} a_{(k)}^{(W)} .
\end{gathered}
$$

With this information at hand, we are now able to apply (4.6) with $g_{4}$ and $\mathcal{F}_{B}^{(1)}, \mathcal{F}_{B}^{(2)}$, yielding two further relationships.

4.3. Deriving the junction conditions. We define the column matrices

$$
E^{(k)}=\left[\begin{array}{llll}
C_{1}^{(k)} & C_{2}^{(k)} & D_{1}^{(k)} & D_{2}^{(k)}
\end{array}\right]^{T}, \quad k=0,1 .
$$

The eight equations obtained in the previous two subsections can then be rewritten as two matrix equations, the first of which is found to be

$$
M E^{(0)}=\left[\begin{array}{c}
\left(\mu_{1} H_{1}+\mu_{2} H_{2}\right) v_{4}^{(0)}\left(x_{B}\right)-\mu_{1} H_{1} v_{2}^{(0)}\left(x_{B}\right)-\mu_{2} H_{2} v_{3}^{(0)}\left(x_{B}\right) \\
\mu_{1} H_{1} C_{1} v_{2}^{(0)}\left(x_{B}\right)+\mu_{2} H_{2} C_{2} v_{3}^{(0)}\left(x_{B}\right) \\
\kappa a_{0}^{(\mathcal{Y})} a_{0}^{(W)}
\end{array}\right],
$$

where $M$ is the $4 \times 4$ matrix

$$
\left[\begin{array}{cccc}
\mu_{1} H_{1} & \mu_{2} H_{2} & 0 & 0 \\
0 & 0 & \mu_{1} H_{1} & \mu_{2} H_{2} \\
\mu_{1} H_{1} D_{1} & \mu_{2} H_{2} D_{2} & -\mu_{1} H_{1} C_{1} & -\mu_{2} H_{2} C_{2} \\
& & & 0
\end{array}\right]
$$

Copyright ( ) by SIAM. Unauthorized reproduction of this article is prohibited. 
where $C_{j}$ and $D_{j}$ are the asymptotic constants from the weight function defined in (2.40). The determinant of $M$ is given by $\operatorname{det}(M)=-\mu_{1}^{2} \mu_{2}^{2} H_{1}^{2} H_{2}^{2}\left(C_{1}-C_{2}\right)^{2}<0$. Therefore, for $C_{1}^{(0)}=C_{2}^{(0)}=D_{1}^{(0)}=D_{2}^{(0)}=0$ (that is, for $W$ to vanish far away from the crack tip as we would expect for such a boundary layer), we have that the matrix in the right-hand side of (4.17) must be equal to zero. From this follow the junction conditions

$$
\begin{aligned}
v_{2}^{(0)}\left(x_{B}\right) & =v_{3}^{(0)}\left(x_{B}\right)=v_{4}^{(0)}\left(x_{B}\right), \\
a_{(0)}^{(W)} & =0 .
\end{aligned}
$$

The latter condition (4.20) yields that $W_{B}^{(0)} \equiv 0$. The second matrix equation is

$$
M E^{(1)}=\left[\begin{array}{c}
\left(\mu_{1} H_{1}+\mu_{2} H_{2}\right)\left(v_{4}^{(0)}\right)^{\prime}\left(x_{B}\right)-\mu_{1} H_{1}\left(v_{2}^{(0)}\right)^{\prime}\left(x_{B}\right)-\mu_{2} H_{2}\left(v_{3}^{(0)}\right)^{\prime}\left(x_{B}\right) \\
\left(\mu_{1} H_{1}+\mu_{2} H_{2}\right) v_{4}^{(1)}\left(x_{B}\right)-\mu_{1} H_{1} v_{2}^{(1)}\left(x_{B}\right)-\mu_{2} H_{2} v_{3}^{(1)}\left(x_{B}\right) \\
\mu_{1} H_{1} C_{1} v_{2}^{(1)}\left(x_{B}\right)+\mu_{2} H_{2} C_{2} v_{3}^{(1)}\left(x_{B}\right)-\mu_{1} H_{1} D_{1}\left(v_{2}^{(0)}\right)^{\prime}\left(x_{B}\right)-\mu_{2} H_{2} D_{2}\left(v_{3}^{(0)}\right)^{\prime}\left(x_{B}\right) \\
\kappa a_{0}^{(\mathcal{Y})} a_{1}^{(W)}-\mu_{1} H_{1} C_{1}\left(v_{2}^{(0)}\right)^{\prime}\left(x_{B}\right)-\mu_{2} H_{2} C_{2}\left(v_{3}^{(0)}\right)^{\prime}\left(x_{B}\right)
\end{array}\right],
$$

where $M$ is the matrix given in (4.18). For $C_{1}^{(1)}=C_{2}^{(1)}=D_{1}^{(1)}=D_{2}^{(1)}=0$, the right-hand matrix is again set to zero. Noting that $a_{0}^{(\mathcal{Y})}=\lambda$ (see (2.45)) and that $\mu_{1} H_{1} C_{1}+$ $\mu_{2} H_{2} C_{2}=0$, setting the fourth row of the right-hand side (RHS) matrix to zero then yields that

$$
a_{(1)}^{(W)}=\frac{1}{\kappa \lambda} \Delta\left\{\left(v^{(0)}\right)^{\prime}\right\},
$$

where

$$
\Delta\left\{\left(v^{(0)}\right)^{\prime}\right\}\left(x_{B}\right)=\left(v_{2}^{(0)}\right)^{\prime}\left(x_{B}\right)-\left(v_{3}^{(0)}\right)^{\prime}\left(x_{B}\right) .
$$

The other conditions imply

$$
\begin{aligned}
& v_{2}^{(1)}\left(x_{B}\right)=v_{4}^{(1)}\left(x_{B}\right)-\frac{\mu_{2} H_{2}}{\mu_{1} H_{1}+\mu_{2} H_{2}}\left(\frac{\alpha}{\pi}+\frac{1}{\lambda}\right) \Delta\left\{\left(v^{(0)}\right)^{\prime}\right\}\left(x_{B}\right), \\
& v_{3}^{(1)}\left(x_{B}\right)=v_{4}^{(1)}\left(x_{B}\right)+\frac{\mu_{1} H_{1}}{\mu_{1} H_{1}+\mu_{2} H_{2}}\left(\frac{\alpha}{\pi}+\frac{1}{\lambda}\right) \Delta\left\{\left(v^{(0)}\right)^{\prime}\right\}\left(x_{B}\right)
\end{aligned}
$$

along with the relationship

$$
\left(\mu_{1} H_{1}+\mu_{2} H_{2}\right)\left(v_{4}^{(0)}\right)^{\prime}\left(x_{B}\right)-\mu_{1} H_{1}\left(v_{2}^{(0)}\right)^{\prime}\left(x_{B}\right)-\mu_{2} H_{2}\left(v_{3}^{(0)}\right)^{\prime}\left(x_{B}\right)=0 .
$$

We stress that $\alpha$ and $\lambda$ are functions of $\kappa$, and so expressions (4.24) and (4.25) describe how the junction conditions depend upon the extent of imperfection of the interface. In particular, $(\alpha / \pi+1 / \lambda)$ is a constant that plays a crucial physical role since it defines the proportionality between the displacement jump in the first order approximation and the angle of opening in the zero order approximation. Equation (4.26) complements conditions (4.19) and (4.20) to give full information for the zero order approximation. We later present numerical results for the normalized constant $\alpha_{I}=(\alpha / \pi+$ $1 / \lambda) /\left(H_{1}+H_{2}\right)$. 
The conditions regarding the first order approximation (4.22), (4.24), and (4.25) can be complemented by a further equation in $\left(v_{m}^{(1)}\right)^{\prime}\left(x_{B}\right)$, which follows from the next level of approximation, i.e., taking $N=2$ in (3.8),

$$
\begin{aligned}
& \mu_{1} H_{1}\left(v_{2}^{(1)}\right)^{\prime}\left(x_{B}\right)+\mu_{2} H_{2}\left(v_{3}^{(1)}\right)^{\prime}\left(x_{B}\right)-\left(\mu_{1} H_{1}+\mu_{2} H_{2}\right)\left(v_{4}^{(1)}\right)^{\prime}\left(x_{B}\right) \\
& =\sum_{j=1}^{2} \int_{\Pi_{B}^{(j)}} \frac{\omega^{2}}{c_{j}^{2}} W_{B}^{(0)} \mathrm{d} \Pi_{B}^{(j)},
\end{aligned}
$$

and by our earlier comment that $W_{B}^{(0)} \equiv 0$, the right side of this expression is zero. At this point we would like to comment that taking higher order approximations and evaluating higher order junction conditions is possible but much more advanced. For example, integrals analogous to that on the RHS of the above expression would depend upon $W_{B}^{(1)}$ and boundary layers from higher order approximations, and so would not in general be zero. However, since we focus on thin strips, $\varepsilon$ is small and so terms in $\varepsilon^{2}$ would give significantly less contribution than the lower order approximations. We later comment on the accuracy of the zero order approximation on page 19 by comparing computations against FEM results in a case where $\varepsilon$ is not too small. We would like to underline that the accuracy will increase for smaller $\varepsilon$, but for very small $\varepsilon$, it is no longer possible to obtain finite-element computations.

5. Numerical simulations and discussions. To enable us to compare results with the perfect interface case discussed in [17] effectively, we seek normalized constants. We first seek a normalized representation of $\alpha$. We introduce the notation

$$
H=H_{1}+H_{2}, \quad H_{*}=\frac{H_{1}-H_{2}}{H_{1}+H_{2}}, \quad \mu_{*}=\frac{\mu_{1}-\mu_{2}}{\mu_{1}+\mu_{2}}, \quad \kappa_{*}=\frac{\kappa\left(\mu_{1}+\mu_{2}\right)}{H}, \quad \lambda_{*}=\lambda H,
$$

where $H_{*}, \mu_{*}$, and $\kappa_{*}$ are nondimensional parameters that respectively describe the geometrical, mechanical, and imperfect properties of the problem. $\lambda$ is the constant dependent on $\mu_{j}, H_{j}$, and $\kappa$ defined in (2.27). $\lambda_{*}$ can be expressed in terms of the other dimensionless parameters as

$$
\lambda_{*}^{2}=\frac{8\left(1+\mu_{*} H_{*}\right)}{\kappa_{*}\left(1-\mu_{*}^{2}\right)\left(1-H_{*}^{2}\right)} .
$$

We also introduce the function

$$
\Xi_{* *}(t)=\frac{t}{\lambda_{*}^{2}+t^{2}}\left(t+\frac{2}{\kappa_{*}\left(1+\mu_{*}\right)} \operatorname{coth} \frac{t\left(1+H_{*}\right)}{2}+\frac{2}{\kappa_{*}\left(1-\mu_{*}\right)} \operatorname{coth} \frac{t\left(1-H_{*}\right)}{2}\right),
$$

which satisfies the relationship $\Xi_{* *}(t)=\Xi_{*}\left(\frac{t}{H}\right)$, and so we can write

$$
\alpha=\int_{0}^{\infty} \frac{\ln \Xi_{*}(\xi)}{\xi^{2}} d \xi=\int_{0}^{\infty} \frac{H^{2} \ln \Xi_{* *}(t)}{t^{2}} \frac{d t}{H}=H \int_{0}^{\infty} \frac{\ln \Xi_{* *}(t)}{t^{2}} d t=H \alpha_{*},
$$

where we have defined the nondimensional quantity $\alpha_{*}$. We find through asymptotic analysis that

$$
\frac{\ln \Xi_{* *}(t)}{t^{2}}=\frac{1}{12} \frac{H_{*}^{3} \mu_{*}-H_{*}^{2}-\mu_{*} H_{*}+1}{1+\mu_{*} H_{*}}+O\left(t^{2}\right), \quad t \rightarrow 0
$$

Copyright @ by SIAM. Unauthorized reproduction of this article is prohibited. 
Mishuris, Movchan, and Bercial [17] showed that in the analogous problem to that discussed in this paper with a perfect interface instead of an imperfect interface,

$$
D_{j}=\alpha_{P}\left(H_{1}+H_{2}\right) C_{j},
$$

where

$$
\alpha_{P}=\frac{1}{\pi} \ln \left\{\left(\frac{1+H_{*}}{2}\right)^{\frac{1+H_{*}}{2}}\left(\frac{1-H_{*}}{2}\right)^{\frac{1-H_{*}}{2}}\right\}-\frac{\mu_{*}}{\pi} \int_{0}^{\infty} \frac{H_{*}-\tanh \left(t H_{*}\right) \operatorname{coth}(t)}{\left(\sinh (t)+\mu_{*} \sinh \left(t H_{*}\right)\right) t} d t .
$$

We have demonstrated (see the form of the constants $C_{j}, D_{j}$ in (2.40)) that for the imperfect interface problem,

$$
D_{j}=\alpha_{I}\left(H_{1}+H_{2}\right) C_{j},
$$

where

$$
\alpha_{I}=-\left(\frac{1}{\pi} \int_{0}^{\infty} \frac{\ln \Xi_{* *}(t)}{t^{2}} d t+\frac{1}{\lambda_{*}}\right)
$$

and since small $\kappa_{*}$ correspond to an interface that is "almost perfect," we would expect $\alpha_{I} \rightarrow \alpha_{P}$ as $\kappa_{*} \rightarrow 0$. Figure 5.1 shows a plot of the ratio $\alpha_{I} / \alpha_{P}$ on axes of $\mu_{*}$ against $H_{*}$ for four different values of $\kappa_{*}$. From this it is easily seen that as $\kappa_{*} \rightarrow 0, \alpha_{I} / \alpha_{P}$ gets close to 1 as expected. The behavior of the weight functions near the crack tip are, however, absolutely different, since the problem is singularly perturbed; that is,
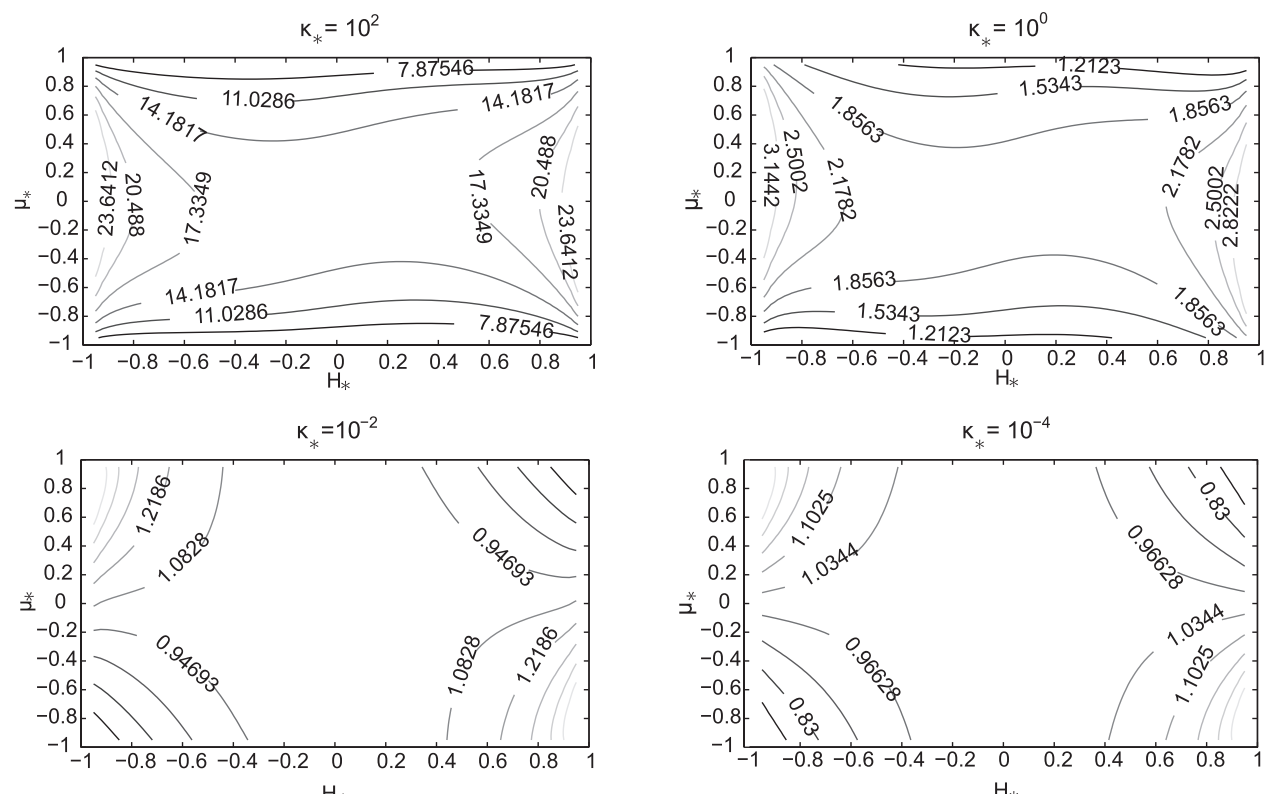

$\mathrm{H}_{*}$

FIG. 5.1. Contour plots of the ratio $\alpha_{I} / \alpha_{P}$ for four different values of $\kappa_{*}$, a dimensionless parameter describing the extent of imperfection of the interface between the two materials. The axes of each plot are $\mu_{*}$ and $H_{*}$, dimensionless parameters respectively describing the mechanical and geometric properties of the problem. The ratio $\alpha_{I} / \alpha_{P}$ gets closer to 1 as $\kappa_{*}$ decreases in value toward 0 .

Copyright $@$ by SIAM. Unauthorized reproduction of this article is prohibited. 


$$
\begin{aligned}
& {[\mathcal{Y}] } \sim \sqrt{\eta \kappa}, \quad \kappa \rightarrow 0, \\
&\left.\mu_{1} \frac{\partial \mathcal{Y}}{\partial Y}\right|_{Y=0+} \sim-\sqrt{\frac{\eta}{\kappa}}, \quad X \rightarrow 0, \quad \kappa \rightarrow 0,
\end{aligned}
$$

where $\eta$ is defined in (2.30). In the plots showing the ratio for small values of $\kappa_{*}$, the highest deviations from 1 occur near the corner of the plot. These correspond to the cases where there is a high contrast between the shear moduli and thicknesses of the two materials. We see that in the cases where the materials have similar shear moduli and thicknesses (nearer the center of the plot), the ratio $\alpha_{I} / \alpha_{P}$ quickly approaches 1 as $\kappa_{*} \rightarrow 0$.

Figure 5.2 shows surface plots of $\alpha_{I}$ on axes of $\mu_{*}$ and $H_{*}$ for $\kappa_{*}=100,1$, and 0.01 . This constant describes the impact that the imperfect interface has upon the junction conditions as described in (4.24) and (4.25). Also shown in the figure is a plot of $\alpha_{P}$. The similarity between the plot of $\alpha_{I}$ for $\kappa_{*}=0.01$ and the plot of $\alpha_{P}$ is evident here. For the cases with larger $\kappa_{*}$ values, we see that $\alpha_{P}$ is differently dependent upon the mechanical and geometric parameters of the problem.

Figure 5.3 shows finite-element plots (COMSOL) of standing wave eigensolutions. For these simulations we use the following geometrical parameters for the elementary cell:
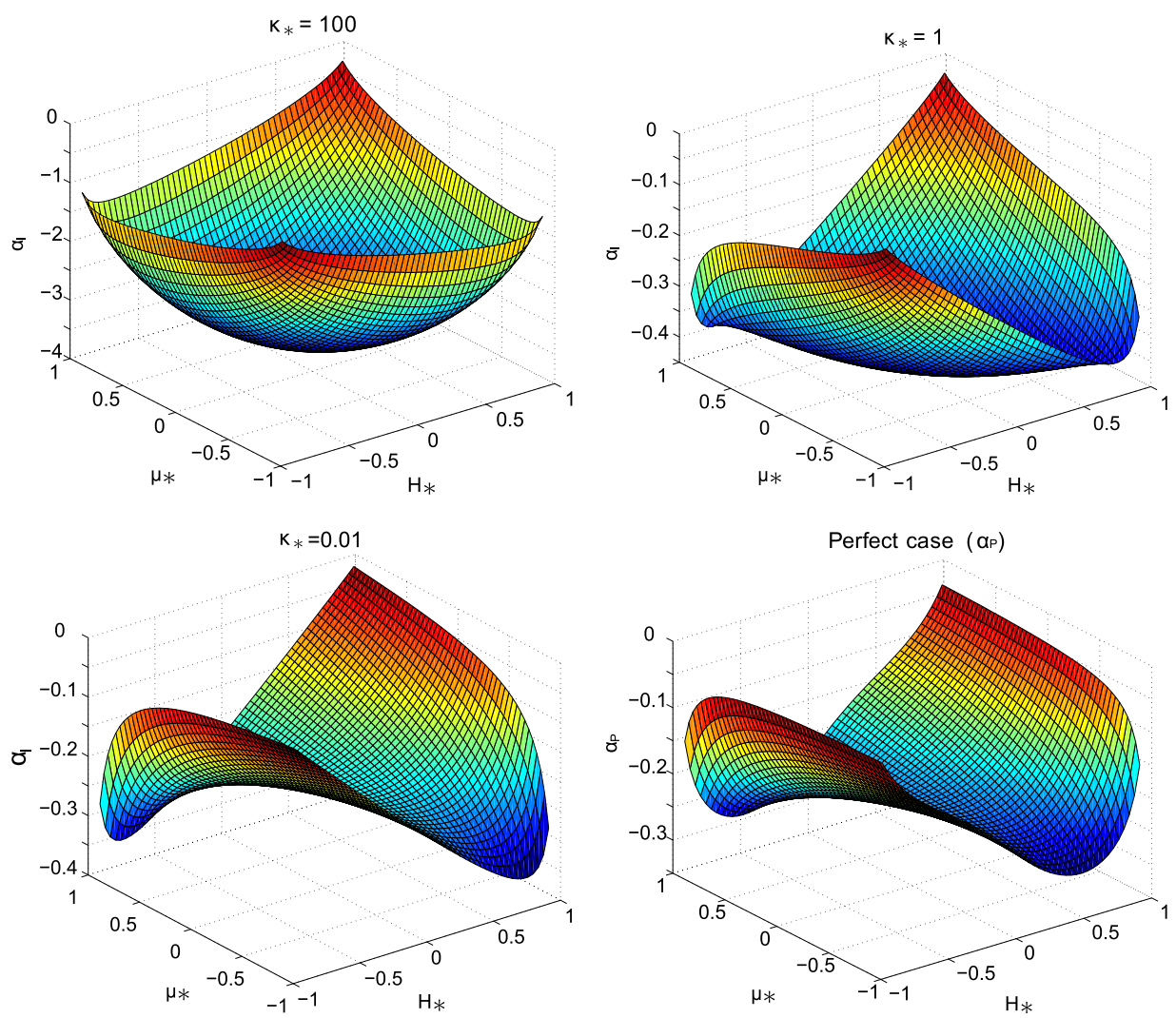

FIG. 5.2. Surface plots of $\alpha_{I}$ for $\kappa_{*}=100,1$, and 0.01 ; also of $\alpha_{P}$, all plotted on axes of $\mu_{*}$ and $H_{*}$. 


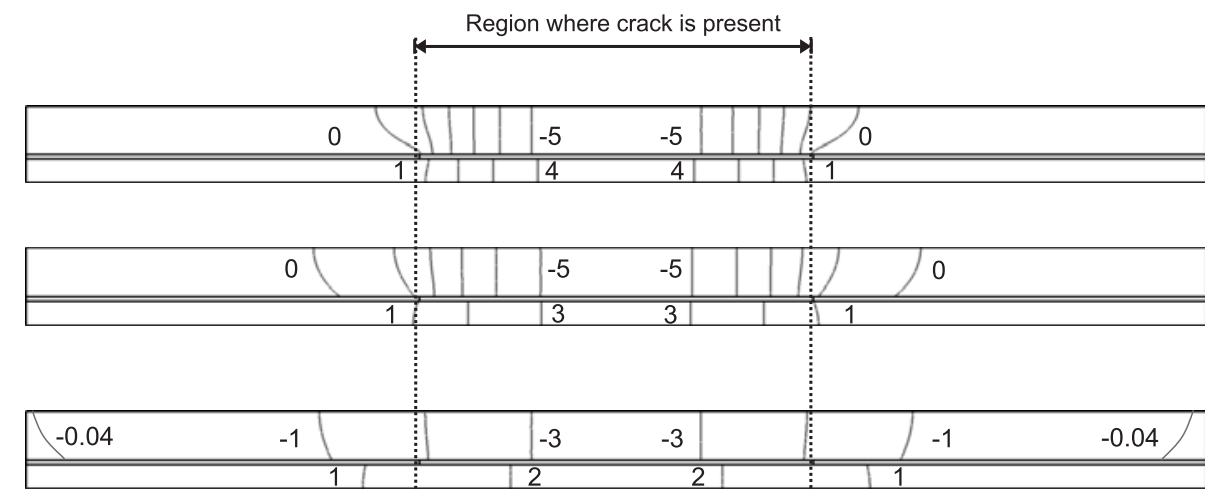

FIG. 5.3. Finite-element computation (COMSOL) contour plot of the eigensolution corresponding to the standing Bloch-Floquet waves for three different values of $\kappa$. Top: Bonding material with shear modulus $1000 \mu_{\text {resin }}$. Middle: Bonding material is epoxy resin. Bottom: Bonding material with shear modulus $\mu_{\text {resin }} / 10$. Contours join points of integer values, and the dotted vertical lines indicate the location of the crack tips.

$$
l=0.8[\mathrm{~m}], \quad a=2.4[\mathrm{~m}], \quad H_{1}=0.1[\mathrm{~m}], \quad H_{2}=0.05[\mathrm{~m}] .
$$

We also use the following material constants that correspond to iron (in $\left.\Pi_{\varepsilon}^{(2)}\right)$ and aluminum $\left(\right.$ in $\Pi_{\varepsilon}^{(1)}$ ):

$$
\begin{aligned}
\mu_{2} & =82 \cdot 10^{9}\left[\mathrm{~N} / \mathrm{m}^{2}\right], \quad \mu_{1}=26 \cdot 10^{9}\left[\mathrm{~N} / \mathrm{m}^{2}\right], \\
\rho_{2} & =7860\left[\mathrm{~kg} / \mathrm{m}^{3}\right], \quad \rho_{1}=2700\left[\mathrm{~kg} / \mathrm{m}^{3}\right] .
\end{aligned}
$$

Presented in this figure are three plots corresponding to Al-Fe strips with different materials bonding them together, with the vertical dotted lines indicating the location of the crack tips. The imperfect interface is modeled in the COMSOL simulations by a thin layer occupied by an adhesive material; this approach was justified in [15], [16], among others. Provided that $h_{\text {resin }} / H_{2}$ is sufficiently small and $\mu_{\text {resin }}$ is small in comparison to $\mu_{1}$ and $\mu_{2}$, this gives $\kappa=h_{\text {resin }} / \mu_{\text {resin }}$.

The second of the three plots in Figure 5.3 uses epoxy resin as the bonding material with parameters

$$
\mu_{\text {resin }}=2.5 \cdot 10^{9}\left[\mathrm{~N} / \mathrm{m}^{2}\right], \quad \rho_{\text {resin }}=1850\left[\mathrm{~kg} / \mathrm{m}^{3}\right], \quad h_{\text {resin }}=0.01[\mathrm{~m}] .
$$

For comparison, the first plot shows a simulation with a gluing layer of shear modulus 1000 greater than that of epoxy resin. The third plot uses a material with shear modulus 10 times less than epoxy resin. Equivalently, these three cases in the top, middle, and bottom parts of the figure correspond to $\kappa_{*}=2.88 \cdot 10^{-3}, \kappa_{*}=2.88$, and $\kappa_{*}=28.8$, respectively. The plots show that the standing wave is more localized and intense in the locality of the crack when the bonding material is stiffer. Conversely, when the bonding material is less stiff, the standing wave extends further beyond the locality of the crack and is less intense. Closely packed contours indicate areas where stress is high; we see that the highest stress is to be found in the vicinity of the crack tip in all three cases. Moreover, as we would expect, the highest stress intensity is found in the case with the stiffest bonding material.

We do not present dispersion diagrams here computed by the asymptotic analysis and COMSOL, as they are similar to those given in [17]. As in that paper, the biggest 
discrepancy between results obtained from asymptotic analysis and numerical simulations appears for the case of the standing waves. In all other situations, the accuracy is very good, with a typical discrepancy between finite-element and asymptotic results of around $0.3 \%$ in the case where the strip has the same dimensions as used throughout this section, which corresponds to $\varepsilon=0.0625$. We remind the reader that we use static boundary layers in the analysis. The standing waves lie in the area of rather high frequencies, which may provide one possible explanation for this phenomenon. This discrepancy needs to be eliminated, and this will form part of a future work.

It is readily seen in the bottom plot of Figure 5.3 (which corresponds to a highly imperfect interface) that the boundary layer support extends almost to the edge of the elementary cell. This extension far away from the crack tips suggests that the boundary layers decay slowly from the crack tips and so may not be assumed independent. In this case, therefore, our analysis may become invalid due to the assumption in our asymptotic procedure that the exponentially decaying boundary layer does not influence the Bloch-Floquet conditions. This assumption is satisfied if $\gamma_{+}$is far from zero, so if $\kappa$ is not too large. More accurately, we assume $\gamma_{+} \gg \frac{\varepsilon}{a-l}$ (see (2.47) for large $\kappa$ ). If the imperfect interface is too weak and this condition is violated, then the junction conditions evaluated here will no longer be accurate and other analysis should be sought.

Appendix A. Derivation of asymptotics of $\boldsymbol{\Xi}_{*}^{+}(\xi)$. We present here the derivation of asymptotics for $\Xi_{*}^{+}(\xi)$. The results of this derivation are used in expressions (2.32) and (2.33). We introduce the auxiliary function

$$
\Theta_{*}^{+}(\xi)=\int_{-\infty-i \beta}^{\infty-i \beta} \frac{\ln \Xi_{*}(t)}{t-\xi} d t
$$

so that $\Xi_{*}^{+}(\xi)=\exp \left((1 / 2 \pi i) \Theta_{*}^{+}(\xi)\right)$ (see $\left.(2.29)\right)$. We first note that $\Theta_{*}^{+}(0)=0$, since the integrand is odd and the second estimate in (2.30) demonstrates integrability of $\boldsymbol{\Xi}_{*}$ at the zero point, allowing us to take $\beta=0$. Thus

$$
\Theta_{*}^{+}(\xi)=\int_{-\infty-i \beta}^{\infty-i \beta}\left[\frac{\ln \Xi_{*}(t)}{t-\xi}-\frac{\ln \Xi_{*}(t)}{t}\right] d t=\xi \int_{-\infty-i \beta}^{\infty-i \beta} \frac{\ln \Xi_{*}(t)}{t(t-\xi)} d t \rightarrow 0, \quad \xi \rightarrow 0,
$$

since the integral is bounded. Also, we have that

$$
\int_{-\infty-i \beta}^{\infty-i \beta} \frac{\ln \Xi_{*}(t)}{t^{2}} d t=\int_{-\infty}^{\infty} \frac{\ln \Xi_{*}(t)}{t^{2}} d t=2 \int_{0}^{\infty} \frac{\ln \Xi_{*}(t)}{t^{2}} d t=2 \alpha
$$

since the integrand is even and again by considering the second equality in (2.30), which indicates that we have integrability at the zero point. Here we have found that

$$
\Theta_{*}^{+}(\xi)=2 \alpha \xi+O\left(|\xi|^{2}\right), \quad \xi \rightarrow 0 .
$$

From this we obtain the following estimate for $\Xi_{*}^{+}(\xi)$ as $\xi \rightarrow 0$ :

$$
\Xi_{*}^{+}(\xi)=1+\frac{\alpha \xi}{\pi i}+O\left(|\xi|^{2}\right), \quad \xi \rightarrow 0 .
$$

We now seek estimates of $\Theta_{*}^{+}(\xi)$ for $\xi \rightarrow \infty$ within the domain. To avoid problems caused by integrating along the real line, we consider $\xi \rightarrow \infty$ in such a way that $\operatorname{Im}(\xi) \rightarrow+\infty$. Integrating (A.1) by parts, splitting the integral in two and manipulating the resulting expression gives 


$$
\Theta_{*}^{+}(\xi)=\int_{0}^{\infty} \ln \left(\frac{1+t / \xi}{1-t / \xi}\right) \frac{\Xi_{*}^{\prime}(t)}{\Xi_{*}(t)} d t
$$

We introduce an arbitrary $R>0$ and split this integral at $R$ to give

$$
\Theta_{*}^{+}(\xi)=\int_{0}^{R} \ln \left(\frac{1+t / \xi}{1-t / \xi}\right) \frac{\Xi_{*}^{\prime}(t)}{\Xi_{*}(t)} d t+\int_{R}^{\infty} \ln \left(\frac{1+t / \xi}{1-t / \xi}\right) \frac{\Xi_{*}^{\prime}(t)}{\Xi_{*}(t)} d t
$$

We then see that

$$
\ln \left(\frac{1+t / \xi}{1-t / \xi}\right)=2 \frac{t}{\xi}+O\left(\frac{t^{3}}{|\xi|^{3}}\right), \quad \xi \rightarrow \infty, \quad 0<t<R,
$$

and from (2.31) we have

$$
\frac{\Xi_{*}^{\prime}(t)}{\Xi_{*}(t)}=\left[-\frac{\mu_{1}+\mu_{2}}{\kappa \mu_{1} \mu_{2}}\right] \frac{1}{t^{2}}+O\left(\frac{1}{t^{3}}\right), \quad t \rightarrow \infty .
$$

This allows us to estimate

$$
\Theta_{*}^{+}(\xi)=\int_{R}^{\infty}\left[-\frac{\left(\mu_{1}+\mu_{2}\right)}{\mu_{1} \mu_{2} \kappa} \frac{1}{t^{2}}+O\left(\frac{1}{t^{3}}\right)\right] \ln \left(\frac{\xi+t}{\xi-t}\right) d t+O\left(\frac{1}{|\xi|}\right), \quad \xi \rightarrow \infty .
$$

After integrating by parts and performing a change of variables, we find that

$$
\int_{R}^{\infty} \frac{1}{t^{2}} \ln \left(\frac{\xi+t}{\xi-t}\right) d t=-\frac{1}{\xi}\left(\ln \left|\frac{1}{\xi^{2}}\right|+i \arg \left(-\frac{1}{\xi^{2}}\right)\right)+O\left(\frac{1}{|\xi|}\right), \quad \xi \rightarrow \infty
$$

and so from (A.9), we deduce that

$$
\Theta_{*}^{+}(\xi)=\frac{2\left(\mu_{1}+\mu_{2}\right)}{\mu_{1} \mu_{2} \kappa \xi} \ln (-i \xi)+O\left(\frac{1}{|\xi|}\right), \quad \operatorname{Im}(\xi) \rightarrow+\infty .
$$

Recalling the relationship between our auxiliary function $\Theta_{*}^{+}$and $\Xi_{*}^{+}$as we discussed after (A.1), we see that

$$
\Xi_{*}^{+}(\xi)=1+\frac{1}{\pi i} \frac{\left(\mu_{1}+\mu_{2}\right)}{\mu_{1} \mu_{2} \kappa} \frac{\ln (-i \xi)}{\xi}+O\left(\frac{1}{|\xi|}\right), \quad \operatorname{Im}(\xi) \rightarrow+\infty .
$$

\section{Appendix B. Theorem.}

THeorem B.1. Let $f(x)$ be the function

$$
f(x)=\frac{1}{2 \pi} \int_{-\infty}^{\infty} \Phi^{+}(t) e^{-i x t} d t
$$

If $\Phi^{+}(t)$ is analytic in $\mathbb{C}^{+}$and

$$
\Phi^{+}(t)=a_{1} t^{-1}+O\left(t^{-(1+\delta)}\right), \quad t \rightarrow \infty,
$$

where $\delta>0$ is small, in the closed half-plane $\overline{\mathbb{C}}^{+}=\mathbb{C}^{+} \cup \mathbb{R}$, then $f(x)=0$ for all $x<0$ and 


$$
\lim _{x \rightarrow 0^{+}} f(x)=-i a_{1} .
$$

Proof. The fact that $f(x)=0$ for all $x<0$ is a direct consequence of the fact that $\Phi^{+}(t)$ is a "+" function. Assume now that $x>0$. From the assumptions on the behavior of the function $\Phi^{+}(t)$, it follows that $\Phi^{+}(t)=a_{1} t^{-1}+R(t)$, where $t R(t) \rightarrow 0$, as $t \rightarrow \infty$, $t \in \overline{\mathbb{C}}^{+}$(including $t \rightarrow \pm \infty, t \in \mathbb{R}$ ).

We write

$$
f(x)=\frac{1}{2 \pi} \lim _{a \rightarrow+\infty}\left\{\int_{a}^{\infty}\left[\Phi^{+}(-t) e^{i x t}+\Phi^{+}(t) e^{-i x t}\right] d t+\int_{-a}^{a} \Phi^{+}(t) e^{-i x t} d t\right\} .
$$

The first integral is

$$
f_{1}(x, a)=\int_{a}^{\infty}\left[\Phi^{+}(-t) e^{i x t}+\Phi^{+}(t) e^{-i x t}\right] d t=f_{11}(x, a)+f_{12}(x, a),
$$

where

$$
f_{11}(x, a)=\int_{a}^{\infty}\left[-\frac{a_{1}}{t} e^{i x t}+\frac{a_{1}}{t} e^{-i x t}\right] d t=-2 i a_{1} \int_{x a}^{\infty} \frac{\sin (t)}{t} d t
$$

and

$$
f_{12}(x, a)=\int_{a}^{\infty}\left[R(-t) e^{i x t}+R(t) e^{-i x t}\right] d t=\int_{x a}^{\infty}\left[\frac{1}{x} R\left(-\frac{t}{x}\right) e^{i t}+\frac{1}{x} R\left(\frac{t}{x}\right) e^{-i t}\right] d t .
$$

Taking $a=x^{-1 / 2}$, we have that $f_{11}\left(x, x^{-1 / 2}\right) \rightarrow-i \pi a_{1}$ and $f_{12}\left(x, x^{-1 / 2}\right) \rightarrow 0$ as $x \rightarrow 0^{+}$. Let us denote the second integral in (B.4) by $f_{2}(x, a)$. Then using analyticity of $\Phi^{+}(t)$ in $\mathbb{C}^{+}$and defining

$$
\Gamma_{a}=\left\{t \in \mathbb{C} \mid t=a e^{i \theta}, 0<\theta<\pi\right\}
$$

we deduce

$$
f_{2}(x, a)=-\int_{\Gamma_{a}} \Phi^{+}(t) e^{-i x t} d t .
$$

We write this in the form

$$
f_{2}(x, a)=f_{21}(x, a)+f_{22}(x, a),
$$

where

$$
f_{21}(x, a)=-\int_{\Gamma_{a}} \frac{a_{1}}{t} e^{-i x t} d t \quad \text { and } \quad f_{22}(x, a)=-\int_{\Gamma_{a}} R(t) e^{-i x t} d t .
$$

Again taking $a=x^{-1 / 2}$, we obtain

$$
f_{21}\left(x, x^{-1 / 2}\right)=-\int_{\Gamma_{x^{-1 / 2}}} \frac{a_{1}}{t} e^{-i x t} d t \sim-a_{1} \int_{\Gamma_{x^{-1 / 2}}} \frac{1}{t} d t=-i \pi a_{1}, \quad x \rightarrow 0^{+} .
$$

Now,

$$
f_{22}(x, a)=-\int_{\Gamma_{a}} R(t) e^{-i x t} d t=-\int_{\Gamma_{x a}} \frac{1}{x} R\left(\frac{t}{x}\right) e^{-i t} d t,
$$

Copyright ( by SIAM. Unauthorized reproduction of this article is prohibited. 
and so $f_{22}\left(x, x^{-1 / 2}\right) \rightarrow 0$ as $x \rightarrow 0^{+}$. By collecting these observations and reconsidering (B.4), we conclude that

$$
f(x) \rightarrow \frac{1}{2 \pi}\left(-i \pi a_{1}-i \pi a_{1}\right)=-i a_{1}, \quad x \rightarrow 0^{+},
$$

which completes the proof.

\section{REFERENCES}

[1] J. D. Achenbach and Z. L. LI, Propagation of horizontally polarized transverse waves in a solid with a periodic distribution of cracks, Wave Motion, 8 (1986), pp. 371-379.

[2] Y. A. Antipov, O. Avila-pozos, S. T. Kolaczkowski, and A. B. Movchan, Mathematical model of delamination cracks on imperfect interfaces, Internat. J. Solids Structures, 38 (2001), pp. 6665-6697.

[3] S. O. Asplund, Structural Mechanics: Classical and Matrix Methods, Prentice-Hall, Englewood Cliffs, NJ, 1966.

[4] O. Avila-pozos and A. B. Movchan, Slow decay of end effects in layered structures with an imperfect interface, J. Engrg. Math., 45 (2003), pp. 155-168.

[5] J.-M. BAIK AND R. B. Thompson, Ultrasonic scattering from imperfect interfaces - A quasi-static model, J. Nondestruct. Eval., 4 (1984), pp. 177-196.

[6] A. Boström and M. Golub, Elastic SH wave propagation in a layered anisotropic plate with interface damage modelled by spring boundary conditions, Quart. J. Mech. Appl. Math., 62 (2009), pp. 39-52.

[7] Y. Benveniste and T. Мiroh, Imperfect soft and stiff interfaces in two-dimensional elasticity, Mech. Mater., 33 (2001), pp. 309-323.

[8] H. F. BuECKNeR, Weight functions and fundamental fields for the penny-shaped and the half-plane crack in three-space, Internat. J. Solids Structures, 23 (1985), pp. 57-93.

[9] M. Golub, C. Zhang, And Y. S. Wang, SH-wave propagation and resonance phenomena in a periodically layered composite structure with a crack, J. Sound Vib., 330 (2011), pp. 3141-3154.

[10] Z. Hashin, Thin interphase/imperfect interface in conduction, J. Appl. Phys., 89 (2001), pp. 2261-2267.

[11] J. W. Hutchinson, M. E. Mear, and J. R. Rice, Crack paralleling an interface between dissimilar materials, J. Appl. Mech. Tech. Phys., 54 (1987), pp. 828-832.

[12] V. Kozlov, V. G. Maz'ya, and A. B. Movchan, Asymptotic Analysis of Fields in Multi-Structures, Oxford University Press, London, 1999.

[13] V. Lazarus AND J. B. LeBlond, Three-dimensional crack-face weight functions for the semi-infinite interface crack-I: Variation of the stress intensity factors due to some small perturbation of the crack front, J. Mech. Phys. Solids, 46 (1998), pp. 489-511.

[14] R. Lipton, Effect of interfacial bonding on Fiber reinforced shafts subject to antiplane shear, Internat. J. Solids Structures, 38 (2001), pp. 369-387.

[15] G. S. MishuRis, Interface crack and nonideal interface concept (Mode III), Int. J. Fract., 107 (2001), pp. 279-296.

[16] G. Mishuris, N. V. Movchan, and A. B. Movchan, Steady-state motion of a Mode-III crack on imperfect interfaces, Quart. J. Mech. Appl. Math., 59 (2006), pp. 487-516.

[17] G. S. Mishuris, A. B Movchan, and J. P. Bercial, Asymptotic analysis of Bloch-Floquet waves in a thin bi-material strip with a periodic array of finite-length cracks, Waves Random Complex Media, 17 (2007), pp. 511-533.

[18] B. Noble, Methods Based on the Wiener-Hopf Technique for the Solution of Partial Differential Equations, Chelsea Publishing Company, New York, 1988.

[19] A. Piccolroaz, G. Mishuris, and A. B. Movchan, Evaluation of the Lazarus-query Leblond constants in the asymptotic model of the interfacial wavy crack, J. Mech. Phys. Solids, 55 (2009), pp. 1575-1600.

[20] A. Piccolroaz, G. Mishuris, and A. B. Movchan, Symmetric and skew-symmetric weight functions in $2 D$ perturbation models for semi-infinite interfacial cracks, J. Mech. Phys. Solids, 57 (2009), pp. $1657-1682$.

[21] J. R. Rice And G. C. Sin, Plane problems of cracks in dissimilar media, J. Appl. Mech. Tech. Phys., 32 (1965), pp. 418-423.

[22] J. R. Willis and A. B. Movchan, Dynamic weight functions for a moving crack. I. Mode I loading, J. Mech. Phys. Solids, 43 (1995), pp. 319-341.

Copyright @ $@$ by SIAM. Unauthorized reproduction of this article is prohibited. 